\title{
Thermal ratchet effects in ferrofluids
}

\author{
Andreas Engel ${ }^{1, *}$ and Peter Reimann ${ }^{2}$ \\ ${ }^{1}$ Institut für Physik, Carl-von-Ossietzky-Universtität, 26111 Oldenburg, Germany, and \\ CNRS-Laboratoire de Physique Théorique, 3 rue de l'Université, 67000 Strasbourg, France. \\ ${ }^{2}$ Theoretische Physik, Universität Bielefeld, 33615 Bielefeld, Germany
}

(Dated: September 27, 2018)

\begin{abstract}
Rotational Brownian motion of colloidal magnetic particles in ferrofluids under the influence of an oscillating external magnetic field is investigated. It is shown that for a suitable time dependence of the magnetic field, a noise induced rotation of the ferromagnetic particles due to rectification of thermal fluctuations takes place. Via viscous coupling, the associated angular momentum is transferred from the magnetic nano-particles to the carrier liquid and can then be measured as macroscopic torque on the fluid sample. A thorough theoretical analysis of the effect in terms of symmetry considerations, analytical approximations, and numerical solutions is given which is in accordance with recent experimental findings.

PACS numbers: 5.40.-a, 82.70.-y, 75.50.Mm
\end{abstract}

\section{INTRODUCTION}

Rotational Brownian motion of colloidal particles is a classical subject in statistical physics [1]. Contrary to its translational cousin, i.e. "usual" Brownian motion, it does not result in changes of the particle location and is hence not as easily demonstrated in experiments. If, however, the suspended particles carry an electric or magnetic moment their orientation couples to external fields and the electric or magnetic relaxation properties of the suspension are direct consequences of the rotational diffusion of the constituting particles. In particular, in the well developed theory of ferrofluids the importance of rotational Brownian motion for the theoretical description of the often complex and surprising hydrodynamic and magnetic properties was recognized already a long time ago [2].

Ferrofluids are suspensions of nano-size ferromagnetic grains in carrier liquids like water or oil. They combine the hydrodynamic properties of Newtonian fluids with the magnetic behaviour of superparamagnets $\underline{3}$. Due to the viscosity of the carrier liquid there is a coupling between the rotation of the magnetic grains and the local vorticity of the hydrodynamic flow. This coupling can, e.g., be used to spin up ferromagnetic drops floating in a non-magnetic fluid of the same density with the help of a rotating magnetic field [4, 5]. Complementary, a rotational ferrofluid flow exposed to a static magnetic field exhibits an enhanced shear viscosity []. . Various related effects such as "negative" rotational viscosity [7], magneto-vortical resonance [8], and anomalously enhanced ac-response due to coherent particle rotation [9] have been investigated. They rely on the exchange of angular momentum between rotating particles and an oscillating magnetic field. In these cases, the imposed non-zero flow vorticity of the ferrofluid is crucial for breaking the symmetry between clockwise and counter-clockwise particle rotation.

In the present paper we investigate a much more indirect and subtle aspect of the interplay between rotational Brownian motion of ferrofluid particles and their relaxation dynamics in an external magnetic field. We will show that a suitably designed time dependent external magnetic field without net rotating component may rectify the fluctuations of the particle orientation and set up a noise-induced rotation of the ferromagnetic grains. We will hence investigate how angular momentum can be transferred from an oscillating magnetic field to a ferrofluid at rest. The effect was predicted theoretically and demonstrated experimentally in a previous short communication [10]. In the present paper we provide much more details on the theoretical description and add several new results.

The extraction of directed motion from random fluctuations is an old and controversial problem in statistical mechanics with a long and interesting history 11, 12, 13, 14]. Although excluded by the second law of thermodynamics for equilibrium systems, rectification of fluctuations is possible in systems driven sufficiently far away from thermal equilibrium [15, 16]. The problem has gained renewed attention under the trademarks of "thermal ratchets" and "Brownian motors" due to its possible relevance for biological transport [17, 18] and the prospects of nano-technology [19, 20, 21].

Ferrofluids are ideal systems to investigate such fluctuation driven transport phenomena and also to demonstrate them experimentally [10]: As already discussed above, the rotational dynamics of the ferromagnetic grains is strongly 
influenced by thermal fluctuations. Appropriate time-dependent potentials can be easily designed with the help of external magnetic fields. Finally, directed rotational transport in ferrofluids should manifest itself as systematic rotation of the ferromagnetic nano-particles. This in turn can be easily detected from the resulting macroscopic torque on the carrier liquid. Various somewhat related but still quite different phenomena in rotational dynamical systems are treated in $22,23,24,25,26]$.

Throughout the paper we will use two basic approximations which simplify the analysis considerably and which are rather common for ferrofluids 2, 3]. The first is to neglect Neel-relaxation of the magnetization, i.e. the rotation of the magnetization vector with respect to the ferromagnetic particle. This is justified for particle sizes that are not too small and amounts to assuming that the magnetic moments are firmly attached to the geometry of the particles. Any reorientation of the magnetic moment hence requires a rotation of the particle as a whole. The second approximation is to neglect dipole-dipole interactions between the particles. Although these interactions may be important in concentrated ferrofluids, they are negligible in sufficiently diluted ones. The ratchet mechanism of central interest in the present investigation can operate without any particle-particle interaction. We will hence assume that our ferrofluids are sufficiently diluted and use a single particle model. For a more quantitative assessment of the role of the dipole-dipole interactions see e.g. 27] and references therein.

The paper is organized as follows. In section @ we introduce the general framework for describing the rotational Brownian motion of a single suspended ferromagnetic particle in an external magnetic field. Section $\amalg$ gives a detailed account of the symmetries characteristic of our system and discusses how these symmetries influence the possibility for a ratchet mechanism to operate. In section IV the central Fokker-Planck equation describing our system is analyzed. Besides an investigation of the weak noise limit we describe the numerical solution of the Fokker-Planck equation and compare the results with two different approximate treatments yielding analytical expressions for the transferred angular momentum. Finally, section $\mathbb{\nabla}$ contains some conclusions.

\section{GENERAL FRAMEWORK}

We consider the rotational motion of a spherical particle of volume $V$ and magnetic moment $\boldsymbol{\mu}$ immersed in a liquid with dynamic viscosity $\eta$ and subject to a horizontal, time dependent, spatially homogeneous magnetic field $\mathbf{H}$. The field is composed of a constant part $H_{x}$ parallel to the $x$-axis and an oscillatory part $H_{y}(t)$ with period $2 \pi / \omega$ along the $y$-direction

$$
\mathbf{H}=\left(H_{x}, H_{y}(t), 0\right) \quad, \quad H_{y}(t+2 \pi / \omega)=H_{y}(t) .
$$

Different choices for the time dependence of $H_{y}$ are of interest. In the present paper we will mainly discuss two cases which are each representative for a whole class. Our first standard choice is

$$
H_{y}(t)=H_{y}^{(1)} \cos (\omega t)+H_{y}^{(2)} \sin (2 \omega t+\delta)
$$

where the amplitudes $H_{y}^{(1,2)}$, and the phase $\delta$ are control parameters. The main features of this time dependence are a zero average over one period and the presence of a higher harmonic of the basic frequency. As a second example, we will also discuss the form

$$
H_{y}(t)=H_{y}^{(0)}+H_{y}^{(1)} \cos (\omega t),
$$

for which the average over one period is different from zero. It arises naturally if the constant field component is not perpendicular to the time dependent one. In any case, the magnetic field is of a pure oscillatory character, i.e. it does not contain a net rotating component.

The orientation of the particle at time $t$ is described by the unit vector $\mathbf{e}(t)=\boldsymbol{\mu}(t) / \mu$ where $\mu$ denotes the modulus of the magnetic moment. The time evolution of $\mathbf{e}$ is given by

$$
\partial_{t} \mathbf{e}=\boldsymbol{\Omega} \times \mathbf{e},
$$

where $\boldsymbol{\Omega}(t)$ denotes the instantaneous angular velocity of the particle. Changes of $\boldsymbol{\Omega}$ are due to torques on the particle. Denoting by $\nabla$ the angular part of the three dimensional Nabla operator, the magnetic torque

$$
\mathbf{N}_{\text {mag }}=-\mathbf{e} \times \nabla U=\mu \mathbf{e} \times \mathbf{H}
$$

derives from the potential energy

$$
U(\mathbf{e}, t)=-\mu \mathbf{e} \cdot \mathbf{H}(t)
$$


of a magnetic dipole in an external field [28]. Further, the viscosity $\eta$ of the carrier liquid gives rise to a viscous torque [29]

$$
\mathbf{N}_{\mathrm{visc}}=-6 \eta V \Omega
$$

Additionally, the interaction between the rotating particle and the surrounding liquid also causes thermal fluctuations which generate a stochastic torque [25]

$$
\mathbf{N}_{\text {stoch }}=\sqrt{12 \eta V k_{B} T} \boldsymbol{\xi}(t)
$$

Here, $\boldsymbol{\xi}(t)$ is a vector of independent, $\delta$-correlated Gaussian noise sources of zero mean, the noise intensity is related to the temperature $T$ and the dissipation (17) of the carrier liquid by the fluctuation-dissipation relation, and $k_{B}$ stands for Boltzmann's constant. Denoting the moment of inertia of the particle by $I$, the equation of motion for $\boldsymbol{\Omega}$ acquires the form

$$
I \partial_{t} \boldsymbol{\Omega}+6 \eta V \boldsymbol{\Omega}=\mu \mathbf{e} \times \mathbf{H}+\sqrt{12 \eta V k_{B} T} \boldsymbol{\xi}(t) .
$$

Eqs.(4) and (9) form a closed set of equations for the description of the rotational motion of the particle. Using experimentally relevant parameter values (density of the particle $\rho \simeq 4 \cdot 10^{3} \mathrm{~kg} / \mathrm{m}^{3}$, particle radius $R \simeq 10 \mathrm{~nm}$, viscosity $\eta \simeq 10^{-1} \ldots 10^{-3}$ Pas, time scale $\Omega / \partial_{t} \Omega \simeq 10^{-4} \mathrm{~s}$ ) we find that the first term on the 1.h.s. of eq. (9) is five to seven orders of magnitude smaller than the second one. We may hence safely neglect inertial effects [2] and find for $\boldsymbol{\Omega}$ in the overdamped limit

$$
\boldsymbol{\Omega}=\frac{\mu}{6 \eta V} \mathbf{e} \times \mathbf{H}+\sqrt{2 D} \boldsymbol{\xi}(t)
$$

where we have introduced $D=k_{B} T / 6 \eta V$. Using this result in (4) yields a closed equation for the time evolution of e

$$
\partial_{t} \mathbf{e}=\frac{\mu}{6 \eta V}(\mathbf{e} \times \mathbf{H}) \times \mathbf{e}+\sqrt{2 D} \boldsymbol{\xi}(t) \times \mathbf{e} .
$$

It is convenient to introduce dimensionless units. To this end we measure time in units of the inverse of the external driving frequency, $t \mapsto t / \omega$, use $6 \eta V \omega / \mu$ as unit for the magnetic field strength, $\mathbf{H} \mapsto(6 \eta V \omega / \mu) \mathbf{H}$, and rescale the noise intensity according to $D \mapsto \omega D$. The evolution equation for the orientation e then reads

$$
\partial_{t} \mathbf{e}=(\mathbf{e} \times \mathbf{H}) \times \mathbf{e}+\sqrt{2 D} \boldsymbol{\xi}(t) \times \mathbf{e} .
$$

Introducing the Brownian relaxation time

$$
\tau_{B}=\frac{3 \eta V}{k_{B} T}
$$

we note that the rescaled, dimensionless noise intensity $D$ occurring in (12) just gives the ratio between the relevant deterministic and stochastic time scales in the system:

$$
D=\frac{k_{B} T}{6 \eta V \omega}=\frac{1}{2 \tau_{B} \omega} .
$$

To proceed, we parametrize the orientation e of the magnetic particle by two angles $\theta$ and $\varphi$ according to

$$
\mathbf{e}=(\sin \theta \cos \varphi, \sin \theta \sin \varphi, \cos \theta)
$$

From (12) we then find the following Langevin equations for the time evolution of these angles [30]

$$
\begin{aligned}
& \partial_{t} \theta=-\partial_{\theta} U+D \cot \theta+\sqrt{2 D} \xi_{\theta}(t) \\
& \partial_{t} \varphi=-\frac{1}{\sin ^{2} \theta} \partial_{\varphi} U+\frac{\sqrt{2 D}}{\sin \theta} \xi_{\varphi}(t) .
\end{aligned}
$$

In the dimensionless units adopted, the noise intensity $D$ is given by (14) and the potential (6) takes the form

$$
U(\theta, \varphi, t)=-\sin \theta\left(H_{x} \cos \varphi+H_{y}(t) \sin \varphi\right)
$$


The thermal fluctuations $\xi_{\theta}(t)$ and $\xi_{\varphi}(t)$ are given by two independent, $\delta$-correlated Gaussian noise sources of zero mean. Note that in (17) we are dealing with multiplicative white noise but since the multiplicative function is independent of $\varphi(t)$, no ambiguity (Ito- vs. Stratonovich-interpretation [31]) arises.

It is instructive also to consider the simplified situation of a one-dimensional dynamics in which the vector $\mathbf{e}$ is assumed to be constrained to the horizontal $x$ - $y$-plane. Accordingly, (16) is replaced by $\theta \equiv \pi / 2$ and (17) simplifies to

$$
\partial_{t} \varphi=F(\varphi, t)+\sqrt{2 D} \xi_{\varphi}(t)
$$

where

$$
F(\varphi, t)=-H_{x} \sin \varphi+H_{y}(t) \cos \varphi
$$

The observable of foremost interest in the present investigation is the time and ensemble averaged torque (5) exerted by the magnetic field upon the magnetic particle in the long time limit, i.e. after initial transient have died out. Since the magnetic field (10) is constrained to the $x$-y plane, only the $z$-component of this magnetic torque can be different from zero. Suppressing the subscript mag in (5) from now on, we denote the averaged $z$-component of the magnetic torque by $\overline{\left\langle N_{z}\right\rangle}$, where $\langle\ldots\rangle$ stands for the ensemble average over the different realizations of the noise terms in (16), (17) and the overbar represents the time average over one period of the magnetic field. Using (11) and (5) we get

$$
\overline{\left\langle N_{z}\right\rangle}=\frac{1}{2 \pi} \int_{0}^{2 \pi} d t\left\langle\sin \theta(t)\left(-H_{x} \sin \varphi(t)+H_{y}(t) \cos \varphi(t)\right)\right\rangle .
$$

Exploiting (18) and (17) one readily finds the equivalent expressions

$$
\overline{\left\langle N_{z}\right\rangle}=\frac{1}{2 \pi} \int_{0}^{2 \pi} d t\left\langle\partial_{\varphi} U(\theta(t), \varphi(t), t)\right\rangle=\frac{1}{2 \pi} \int_{0}^{2 \pi} d t\left\langle\partial_{t} \varphi(t) \sin ^{2} \theta(t)\right\rangle
$$

For reasons of ergodicity, the ensemble average in (21) is equivalent to a time average of a single realization over an infinite time interval. Then the extra time average over one period of the external driving drops out and we are left with

$$
\overline{\left\langle N_{z}\right\rangle}=\lim _{\left(t_{f}-t_{i}\right) \rightarrow \infty} \frac{1}{t_{f}-t_{i}} \int_{t_{i}}^{t_{f}} d t \partial_{t} \varphi(t) \sin ^{2} \theta(t)
$$

and similarly for the equivalent expressions in (21), (22).

In the absence of thermal fluctuations, the particle orientation is governed by the overdamped deterministic relaxation dynamics given by (16), (17) with $D=0$. Hence, at any given moment, the orientation $\mathbf{e}(t)$ tends to align with the instantaneous magnetic field $\mathbf{H}(t)$ which lies in the $x$ - $y$-plane. One can then show 32 that $\theta(t)$ converges to $\pi / 2$ for $t \rightarrow \infty$ and that $\varphi(t)$ approaches a periodic long time behaviour. This implies via (23) that

$$
\overline{\left\langle N_{z}\right\rangle}=\lim _{\left(t_{f}-t_{i}\right) \rightarrow \infty} \frac{\varphi\left(t_{f}\right)-\varphi\left(t_{i}\right)}{t_{f}-t_{i}}=0
$$

i.e., in the absence of thermal fluctuations no particle rotation will occur and no average torque can arise. An explicit example is displayed in Fig. 1]

This scenario changes fundamentally in the presence of fluctuations, i.e. if $D \neq 0$. As shown qualitatively in Fig. 1 for small noise intensities, the time dependence of $\varphi(t)$ and $\theta(t)$ will still closely follow the deterministic trajectories, except that now also rare, fluctuation induced transitions between different deterministic solutions may occur. If the rates of forward (increasing $\varphi$ by $2 \pi$ ) and backward transitions (decreasing $\varphi$ by $2 \pi$ ) are different from each other, then on the average a net rotation of the particle will occur, implying with (24) that $\overline{\left\langle N_{z}\right\rangle} \neq 0$. This is a manifestation of the so-called ratchet effect [15] in which an unbiased potential and undirected Brownian fluctuations cooperate to produce directed transport. The detailed operation of the ratchet mechanism for the rotational motion of a colloidal particle is the main focus of the present paper. 


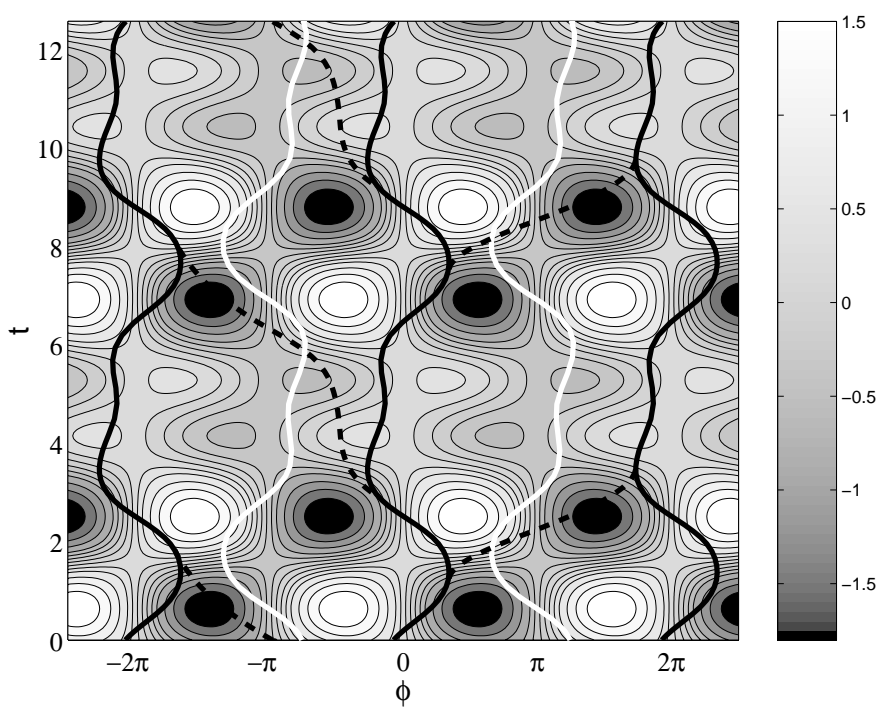

FIG. 1: Space-time plot of the potential $U(\theta=\pi / 2, \varphi, t)$ from eq. (18) for the time dependence (2) with $H_{x}=0.3, H_{y}^{(1)}=$ $H_{y}^{(2)}=1$, and $\delta=0$. Dark and bright regions correspond to small and large values of $U$, respectively. In the long-time limit, the deterministic dynamics (16), (17) with $D=0$ approaches $\theta(t)=\pi / 2$ and a periodical $\varphi(t)$, oscillating back and forth as represented by either of the full black lines. In the presence of a small amount of noise, occasional transitions across the unstable deterministic orbits shown as full white lines become possible which are schematically indicated by the dashed lines. The spatial asymmetry and temporal anharmonicity of the potential conspire to yield slightly different rates for noise induced increments and decrements of $\varphi$, respectively. As a result a noise driven rotation of the particles arises. For a detailed discussion see section IVA

\section{SYMMETRIES}

Symmetry considerations admit conclusions from the equations of motion without actually solving them. For the investigation of ratchet systems, studies of symmetry turned out to be valuable since directed transport generically occurs if it is not forbidden by symmetries, a statement referred to as Curie's principle 15, 33, 34, 35, 36, 37, 38, 39, 40, 41, 42, 43]. Here we specifically address the question which transformations of the magnetic field components $H_{x}$ and $H_{y}(t)$ in (1) leave the average $z$-component of the torque invariant, $\overline{\left\langle N_{z}\right\rangle} \mapsto \overline{\left\langle N_{z}\right\rangle}$, and which lead to its inversion, $\overline{\left\langle N_{z}\right\rangle} \mapsto-\overline{\left\langle N_{z}\right\rangle}$.

From Eq. (23) one readily concludes that the following transformations of $\varphi$ and $\theta$ imply the indicated transformation behavior of $\overline{\left\langle N_{z}\right\rangle}$ :

$$
\begin{aligned}
& \varphi(t) \mapsto \varphi(t)+\Delta \varphi \quad \text { and } \quad \theta(t) \mapsto \theta(t) \quad \text { implies } \quad \overline{\left\langle N_{z}\right\rangle} \mapsto \overline{\left\langle N_{z}\right\rangle} \\
& \varphi(t) \mapsto-\varphi(t)+\Delta \varphi \quad \text { and } \quad \theta(t) \mapsto \theta(t) \quad \text { implies } \quad \overline{\left\langle N_{z}\right\rangle} \mapsto-\overline{\left\langle N_{z}\right\rangle} \\
& \varphi(t) \mapsto \varphi(t) \\
& \text { and } \quad \theta(t) \mapsto-\theta(t)+\pi \quad \text { implies } \\
& \overline{\left\langle N_{z}\right\rangle} \mapsto \overline{\left\langle N_{z}\right\rangle} \\
& \varphi(t) \mapsto \varphi(t+\Delta t) \\
& \text { and } \quad \theta(t) \mapsto \theta(t+\Delta t) \\
& \text { implies } \\
& \overline{\left\langle N_{z}\right\rangle} \mapsto \overline{\left\langle N_{z}\right\rangle} \\
& \varphi(t) \mapsto \varphi(-t) \\
& \text { and } \quad \theta(t) \mapsto \theta(-t) \\
& \text { implies } \\
& \overline{\left\langle N_{z}\right\rangle} \mapsto-\overline{\left\langle N_{z}\right\rangle}
\end{aligned}
$$

where $\Delta \varphi$ and $\Delta t$ are arbitrary. Observing that the values of both the original and the transformed $\theta(t)$ are restricted to the interval $[0, \pi]$ and that the entire interval will indeed be sampled by some realization of the dynamics (16), (17), it follows that no transformations of the form $\theta(t) \mapsto \theta(t)+\Delta \theta$ with $\Delta \theta \neq 0$ or $\theta(t) \mapsto-\theta(t)+\Delta \theta$ with $\Delta \theta \neq \pi$ are possible. Finally, in order to conclude $\overline{\left\langle N_{z}\right\rangle} \mapsto-\overline{\left\langle N_{z}\right\rangle}$ in (29) one has to exploit that in (23) the limit $\left(t_{f}-t_{i}\right) \rightarrow \infty$ is not necessarily tantamount to $t_{f} \rightarrow \infty$ with $t_{i}$ kept fixed, but can also be realized by letting $t_{i} \rightarrow-\infty$ with $t_{f}$ kept fixed.

Closer inspection of (23) reveals that any further symmetry transformation that leaves $\overline{\left\langle N_{z}\right\rangle}$ invariant or changes its sign can be composed of the "elementary" transformations (25)-(29).

Next we ask ourselves what equations of motion govern the transformed $\theta(t), \varphi(t)$ defined by (25)-(29). In the simplest case (25) one readily concludes from (16) and (17) that the transformed $\theta(t), \varphi(t)$ satisfy the same equations of motion (16) and (17) but with a transformed potential $U(\theta, \varphi, t) \mapsto U(\theta, \varphi-\Delta \varphi, t)+\Delta U$ with arbitrary $\Delta U$. 
Turning to the second transformation (26) one finds similarly that the transformed $\theta(t)$ and $\varphi(t)$ satisfy the equations of motion (16) and (17) but now with a transformed potential $U(\theta, \varphi, t) \mapsto U(\theta,-\varphi+\Delta, t)+\Delta U$ and in addition with a transformed noise $\xi_{\varphi}(t) \mapsto-\xi_{\varphi}(t)$. Since the statistical properties of the two noises $-\xi_{\varphi}(t)$ and $\xi_{\varphi}(t)$ are equal, i.e. they are identical stochastic processes, the same follows for the corresponding processes given by (16) and (17). In other words, to each realization of the original $\theta(t), \varphi(t)$ corresponds a realization of the transformed $\theta(t), \varphi(t)$ occurring with the same probability, and vice versa. Since the average torque in (23) is independent of the specific realization $\theta(t), \varphi(t)$ with probability 1 (i.e. up to a set of realizations of measure zero) we can conclude that in this case $\overline{\left\langle N_{z}\right\rangle} \mapsto-\overline{\left\langle N_{z}\right\rangle}$. Similar considerations can be done for the cases given by (27) and (28). The last transformation (29) is insofar special as a mapping between the dynamics of the original and the transformed variables is possible only, if the term $D \cot \theta(t)$ in (16) identically vanishes, i.e. if $\theta(t) \equiv \pi / 2$. We therefore find the following implications of transformations in the potential $U(\theta, \varphi, t)$ :

$$
\begin{aligned}
& U(\theta, \varphi, t) \mapsto U(\theta, \varphi-\Delta \varphi, t)+\Delta U \quad \text { implies } \quad \overline{\left\langle N_{z}\right\rangle} \mapsto \overline{\left\langle N_{z}\right\rangle} \\
& U(\theta, \varphi, t) \mapsto U(\theta,-\varphi+\Delta \varphi, t)+\Delta U \quad \text { implies } \quad \overline{\left\langle N_{z}\right\rangle} \mapsto-\overline{\left\langle N_{z}\right\rangle} \\
& U(\theta, \varphi, t) \mapsto U(-\theta+\pi, \varphi, t)+\Delta U \quad \text { implies } \quad \overline{\left\langle N_{z}\right\rangle} \mapsto \overline{\left\langle N_{z}\right\rangle} \\
& U(\theta, \varphi, t) \mapsto U(\theta, \varphi, t+\Delta t)+\Delta U \quad \text { implies } \quad \overline{\left\langle N_{z}\right\rangle} \mapsto \overline{\left\langle N_{z}\right\rangle} \\
& U(\theta, \varphi, t) \mapsto-U(\theta, \varphi,-t)+\Delta U \quad \text { implies } \quad \overline{\left\langle N_{z}\right\rangle} \mapsto-\overline{\left\langle N_{z}\right\rangle} \quad \text { (provided } \theta(t) \equiv \pi / 2 \text { ), }
\end{aligned}
$$

where $\Delta U$ is an arbitrary constant.

Finally, we have to determine all those transformations of the magnetic field components $H_{x}$ and $H_{y}(t)$ in (1) which correspond via (18) to transformations of the potential $U(\theta, \varphi, t)$ given in (30)-(34). Since changes in $H_{x}$ and $H_{y}(t)$ always imply changes in the potential $U$ that depend on $\theta$ and $\varphi$, we can only induce transformations with $\Delta U=0$. The only non-trivial way to realize (30) is $\left(H_{x}, H_{y}(t)\right) \mapsto\left(-H_{x},-H_{y}(t)\right)$ combined with $\Delta \varphi=(2 n+1) \pi$ with an arbitrary integer $n$. Similarly, (31) and (33) are equivalent to $\left(H_{x}, H_{y}(t)\right) \mapsto\left(H_{x},-H_{y}(t)\right)$ and $\left(H_{x}, H_{y}(t)\right) \mapsto$ $\left(H_{x}, H_{y}(t+\Delta t)\right)$. On the other hand (32) only admits the trivial realization $\left(H_{x}, H_{y}(t)\right) \mapsto\left(H_{x}, H_{y}(t)\right)$. Finally, (34) can be implemented by $\left(H_{x}, H_{y}(t)\right) \mapsto\left(-H_{x},-H_{y}(-t)\right)$. In summary, we find that

$$
\begin{aligned}
& \left(H_{x}, H_{y}(t)\right) \mapsto\left(-H_{x},-H_{y}(t)\right) \quad \text { implies } \quad \overline{\left\langle N_{z}\right\rangle} \mapsto \overline{\left\langle N_{z}\right\rangle} \\
& \left(H_{x}, H_{y}(t)\right) \mapsto\left(H_{x},-H_{y}(t)\right) \quad \text { implies } \quad \overline{\left\langle N_{z}\right\rangle} \mapsto-\overline{\left\langle N_{z}\right\rangle} \\
& \left(H_{x}, H_{y}(t)\right) \mapsto\left(H_{x}, H_{y}(t)\right) \quad \text { implies } \quad \overline{\left\langle N_{z}\right\rangle} \mapsto \overline{\left\langle N_{z}\right\rangle} \\
& \left(H_{x}, H_{y}(t)\right) \mapsto\left(H_{x}, H_{y}(t+\Delta t)\right) \quad \text { implies } \quad \overline{\left\langle N_{z}\right\rangle} \mapsto \overline{\left\langle N_{z}\right\rangle} \\
& \left(H_{x}, H_{y}(t)\right) \mapsto\left(-H_{x},-H_{y}(-t)\right) \quad \text { implies } \quad \overline{\left\langle N_{z}\right\rangle} \mapsto-\overline{\left\langle N_{z}\right\rangle} \quad \text { (provided } \theta(t) \equiv \pi / 2 \text { ), }
\end{aligned}
$$

where the trivial result (37) is listed only for the sake of completeness.

While (35)-(38) are intuitively more or less obvious, (39) respectively (34) is not. The additional condition $\theta(t) \equiv \pi / 2$ shows that the constrained dynamics (19), (20) has an extra symmetry which is lost if the full two-dimensional dynamics (16), (17) is considered.

We note that (35)-(39) could also have been obtained in a more direct way. Our somewhat more involved line of reasoning has the advantage that we can exclude that there are any transformations other than (35)-(39) and combinations thereof which would leave $\overline{\left\langle N_{z}\right\rangle}$ invariant or change its sign.

In order to see that the above considerations already allow some non-trivial conclusions about the possibility of angular momentum transfer from the oscillating magnetic field to the particle we combine (35) and (36), (36) and (38), and (35) and (39) to obtain

$$
\begin{aligned}
& \left(H_{x}, H_{y}(t)\right) \mapsto\left(-H_{x}, H_{y}(t)\right) \quad \text { implies } \quad \overline{\left\langle N_{z}\right\rangle} \mapsto-\overline{\left\langle N_{z}\right\rangle} \\
& \left(H_{x}, H_{y}(t)\right) \mapsto\left(H_{x},-H_{y}(t+\Delta t)\right) \quad \text { implies } \quad \overline{\left\langle N_{z}\right\rangle} \mapsto-\overline{\left\langle N_{z}\right\rangle} \\
& \left(H_{x}, H_{y}(t)\right) \mapsto\left(H_{x}, H_{y}(-t)\right) \quad \text { implies } \quad \overline{\left\langle N_{z}\right\rangle} \mapsto-\overline{\left\langle N_{z}\right\rangle} \quad \text { (provided } \theta(t) \equiv \pi / 2 \text { ) }
\end{aligned}
$$

respectively. From (40) we immediately can infer that the constant field in $x$-direction is indispensable for a non-zero average torque,

$$
H_{x}=0 \Rightarrow \overline{\left\langle N_{z}\right\rangle}=0
$$

Similarly (411) implies that for a time dependence obeying $H_{y}(t)=-H_{y}(t+\Delta t)$ for some $\Delta t$ the average torque has to vanish as well

$$
H_{y}(t)=-H_{y}(t+\Delta t) \Rightarrow \overline{\left\langle N_{z}\right\rangle}=0
$$


Choosing in particular $\Delta t=\pi$, i.e. half the period of the external driving, we realize that an oscillating field $H_{y}(t)$ with a Fourier expansion containing only odd multiples of the basic frequency

$$
H_{y}(t)=\sum_{n=1,3,5, \ldots} H_{y}^{(n)} \cos \left(n t+\delta_{n}\right)
$$

will result in a zero average torque $\overline{\left\langle N_{z}\right\rangle}$, irrespective of the particular choices of the amplitudes $H_{y}^{(n)}$ and phases $\delta_{n}$. Eqs.(43) and (45) motivate our choices (2) and (3) as simple time dependencies of the oscillating field resulting in a non-zero average torque $\overline{\left\langle N_{z}\right\rangle}$.

For the special time dependence (2) we find from (41)

$$
\overline{\left\langle N_{z}\right\rangle}(\delta+\pi)=-\overline{\left\langle N_{z}\right\rangle}(\delta)
$$

As a consequence, upon continuously varying $\delta$ we can infer that there must exist a $\delta_{0} \in[0, \pi)$ such that $\overline{\left\langle N_{z}\right\rangle}=0$ for $\delta=\delta_{0}$ or $\delta=\delta_{0}+\pi$. It is important to note that this case of zero averaged torque is qualitatively different from the situations described by eqs. (43) and (44) since it is due to the fine tuning of a parameter rather than resulting from an underlying symmetry. Consequently, upon variation of $\delta$ around $\delta_{0}$ the average torque $\overline{\left\langle N_{z}\right\rangle}$ changes sign, which is a realization of a so-called current inversion [15]. Furthermore, by fixing $\delta=\delta_{0}$, a sign change of $\overline{\left\langle N_{z}\right\rangle}$ will generically also occur upon variation of any other parameter of the system. Interesting examples for such a parameter include the driving frequency $\omega$, the noise intensity $D$, and the particle volume $V$. In the latter case we hence face the intriguing possibility that in a polydisperse ferrofluid under the same experimental conditions the larger and smaller particles will rotate in opposite directions.

We finally note that in the case where the dynamics is constrained to the $x$-y-axis, the additional symmetry (42) implies that $\delta_{0}=\pi / 2$. Consequently the $\delta$-values where the torque changes sign are fixed by the additional symmetry and do not depend on the other parameters of the problem. Hence no current reversal upon changes in these parameters is possible. This is the main difference between the full two-dimensional dynamics (16), (17) and its simplified one-dimensional counterpart (19), see also 37, 44, 45, 46].

\section{ANALYSIS OF THE FOKKER-PLANCK EQUATION}

A quantitative analysis of the overdamped Brownian rotation of a single ferrofluid particle in a time dependent external field can be built on the numerical simulation of the Langevin equations (16), (17). Such simulations unambiguously show the occurrence of noise induced rotation of the particle in an oscillating field [30]. For a detailed

study of the influence of the various parameters of the problem on the transferred torque $\overline{\left\langle N_{z}\right\rangle}$ it is, however, more convenient to start with the equivalent Fokker-Planck Equation (FPE) for the probability density $P=P(\theta, \varphi, t)$ for the orientation (15) of the particle

$$
\begin{aligned}
\partial_{t} P & =\nabla(P \nabla U)+D \nabla^{2} P \\
& =\frac{1}{\sin \theta} \partial_{\theta}\left(\sin \theta P \partial_{\theta} U\right)+\frac{1}{\sin ^{2} \theta} \partial_{\varphi}\left(P \partial_{\varphi} U\right)+\frac{D}{\sin \theta} \partial_{\theta}\left(\sin \theta \partial_{\theta} P\right)+\frac{D}{\sin ^{2} \theta} \partial_{\varphi}^{2} P .
\end{aligned}
$$

Here $\nabla$ denotes the angular part of the three dimensional Nabla operator. The equivalence of (16), (17) and (47) is, e.g., shown in [30]. For a time-periodic potential $U(\theta, \varphi, t)$, the solution $P(\theta, \varphi, t)$ of (47) will also be periodic after initial transients have died out. From this solution we can determine the average orientation of the particle

$$
\langle\mathbf{e}(t)\rangle=\int_{0}^{2 \pi} d \varphi \int_{0}^{\pi} d \theta \sin \theta(\sin \theta \cos \varphi, \sin \theta \sin \varphi, \cos \theta) P(\theta, \varphi, t)
$$

with the help of which the average torque from (21)-(22) can be calculated according to (51) in yet another way, namely

$$
\overline{\left\langle N_{z}\right\rangle}=\frac{1}{2 \pi} \int_{0}^{2 \pi} d t[\langle\mathbf{e}(t)\rangle \times \mathbf{H}(t)]_{z} .
$$

In the present section we discuss the numerical and approximate analytical solution of the Fokker-Planck equation (47) and the corresponding results (48), (49) for the averaged torque $\overline{\left\langle N_{z}\right\rangle}$. To first substantiate the intuitive understanding of the ratchet effect in our system advocated with Fig. 1 we start by considering the weak noise limit $D \rightarrow 0$. To keep the analysis simple we will restrict ourselves in this part to the simplified one-dimensional model defined by (19), (20). In the second subsection we detail the numerical methods used to solve the FPE for the general case. Finally we present some analytical results from the perturbative solution of the FPE. 


\section{A. Instantons in $d=1$}

Our starting point is the simplified one-dimensional model dynamics (19), (20) with a $\delta$-correlated, unbiased Gaussian noise source $\xi_{\varphi}(t)$. The deterministic dynamics described by (19) with $D=0$ has a family of stable and unstable periodic orbits $\varphi_{\mathrm{s}}(t) \pm 2 \pi n$ and $\varphi_{\mathrm{us}}(t) \pm 2 \pi n$, respectively, where $n$ is an arbitrary integer (cf. Fig. 11) 32]. Hence deterministically the particle simply follows the direction of the magnetic field with a certain time lag due to viscous damping and no particle rotation can occur.

For any non-zero noise intensity $D>0$, there will be few stochastic transitions between the deterministic trajectories and their detailed form in the limit $D \rightarrow 0$ can be determined. Comparing the associated rates for forward (increasing $\varphi$ by $2 \pi$ ) and backward (decreasing $\varphi$ by $2 \pi$ ) phase slips we may directly obtain direction and magnitude of the noise-driven transport by means of (24).

The analysis of this section builds on the path integral representation for the transition probability of a Markovian stochastic process [47, 48, 49]. The main complication is the time dependence of the potential $U(\theta, \varphi, t)$. In notation and general strategy we follow the recent detailed analysis of noise-driven escape over oscillating barriers [50]. We will only determine the dominating exponential term in the transition probabilities. A more detailed analysis including also the prefactor can be done along the lines of ref. [50].

The transition probability $p\left(\varphi_{f}, t_{f} \mid \varphi_{i}, t_{i}\right)$ of the Markov process described by (19) can be written in the form

$$
p\left(\varphi_{f}, t_{f} \mid \varphi_{i}, t_{i}\right)=\int_{\varphi\left(t_{i}\right)=\varphi_{i}}^{\varphi\left(t_{f}\right)=\varphi_{f}} \mathcal{D} \varphi(\cdot) \exp \left(-\frac{S[\varphi(\cdot)]}{D}\right),
$$

where the action functional $S[\varphi(\cdot)]$ is given by

$$
S[\varphi(\cdot)]=\frac{1}{4} \int_{t_{i}}^{t_{f}} d t\left(\partial_{t} \varphi(t)-F(\varphi(t), t)\right)^{2} .
$$

with $F(\varphi, t)$ defined in (20). The intuitive understanding behind this representation is that all possible trajectories $\varphi(t)$ starting at initial time $t_{i}$ in $\varphi_{i}$ and arriving at final time $t_{f}$ in $\varphi_{f}$ contribute to the transition probability $p\left(\varphi_{f}, t_{f} \mid \varphi_{i}, t_{i}\right)$, each with a weight related to the value of the action $S$ evaluated along this trajectory. In the weak noise limit $D \rightarrow 0$ a Laplace argument tells us that only those transitions $\varphi^{*}(t)$ contribute significantly to $p\left(\varphi_{f}, t_{f} \mid \varphi_{i}, t_{i}\right)$ for which the action is minimal. All others have probabilities which are exponentially small for $D \rightarrow 0$. In the weak noise limit we are hence able to determine the precise form of the (dominating) stochastic transitions by solving a variational problem.

Under rather general conditions which are satisfied in our particular setting one can show that the transition rate between two stable orbits of the dynamics is determined by the probability to reach from the starting stable orbit the unstable orbit separating the two stable ones [50]. The subsequent relaxation to the final stable orbit is then purely deterministic and does not contribute to the transition probability $p\left(\varphi_{f}, t_{f} \mid \varphi_{i}, t_{i}\right)$ in the weak-noise limit.

We have hence to solve a variational problem for which the initial and final point of the trajectory are not fixed. Instead they are required to lie on two known functions $\varphi_{\mathrm{S}}(t)$ and $\varphi_{\mathrm{us}}(t)$, respectively. If we perform the optimization with respect to these locations we find

$$
\partial_{t} \varphi^{*}\left(t_{i}\right)=F\left(\varphi^{*}\left(t_{i}\right), t_{i}\right) \quad \text { and } \quad \partial_{t} \varphi^{*}\left(t_{f}\right)=F\left(\varphi^{*}\left(t_{f}\right), t_{f}\right) .
$$

Hence the optimal transition trajectory $\varphi^{*}(t)$ must be parallel to the stable and unstable orbit at the contact points. This in turn implies $t_{i}=-\infty$ and $t_{f}=\infty$. The optimal transition trajectory $\varphi^{*}(t)$ hence starts out at $t_{i}=-\infty$ at the stable orbit, moves for a very long time near to it, changes then in a rather short time interval to the immediate neighbourhood of the unstable orbit (hence the name "instanton") which it finally reaches at $t_{f}=\infty[50$ ].

From the minimization of the action $S[\varphi(\cdot)]$ with respect to the functions $\varphi(t)$ we get the usual Euler-Lagrange equation for the minimizing instanton $\varphi^{*}(t)$ which for the action given by (51) takes the form

$$
\partial_{t}^{2} \varphi^{*}(t)=\partial_{t} F\left(\varphi^{*}(t), t\right)+F\left(\varphi^{*}(t), t\right) \partial_{\varphi} F\left(\varphi^{*}(t), t\right) .
$$

Choosing for the time dependence of $H_{y}(t)$ the example specified by (2) we have solved (53) numerically as a boundary value problem for a system of ordinary differential equations. The initial and final points were chosen slightly off the stable and unstable orbit, respectively. If the difference between the initial and final time, $t_{f}-t_{i}$, is larger than several periods of the external driving, the solution hardly depends on the precise value of these deviations from the deterministic orbits. Having obtained $\varphi^{*}(t)$, the corresponding value of the action $S$ can be calculated from (51). 

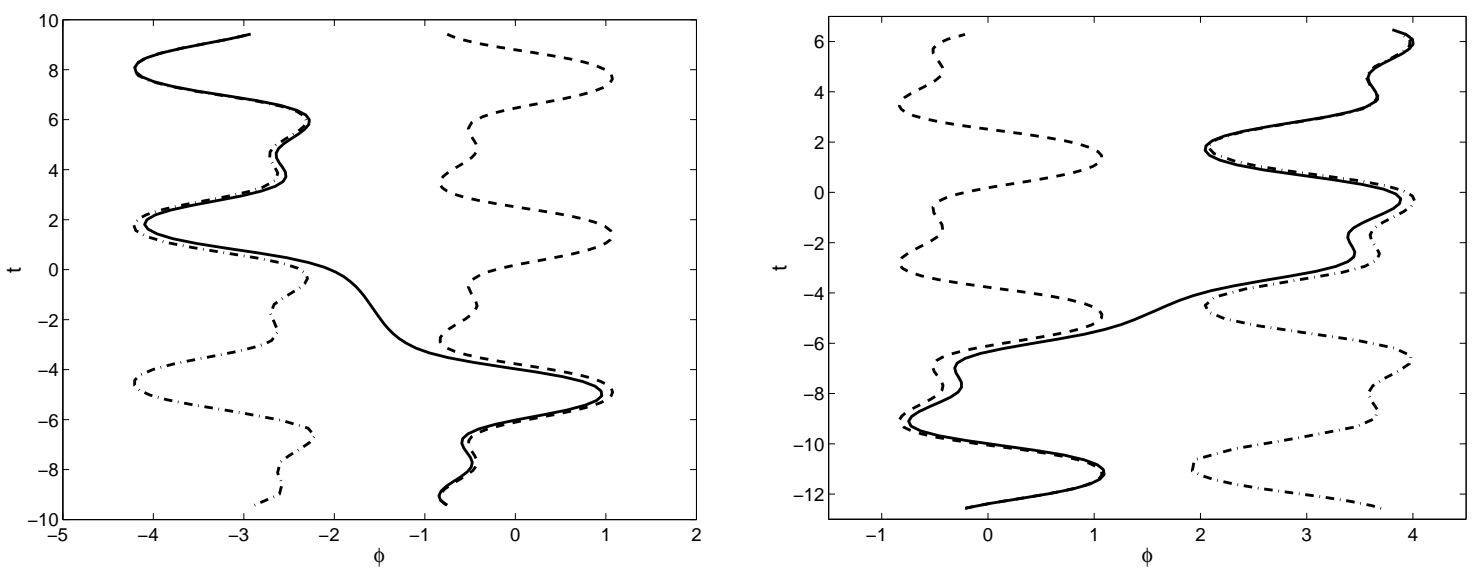

FIG. 2: Numericaly determined instanton trajectories (full lines) describing the dominating weak noise stochastic transitions between stable (dashed lines) and unstable (dashed-dotted lines) deterministic periodic orbits of the one-dimensional rotational dynamics of a ferrofluid particle described by (19), (20), (2). The parameter values are $H_{x}=0.3, H_{y}^{(1)}=H_{y}^{(2)}=1$ and $\delta=0$. For the forward instanton, increasing $\varphi$ by $2 \pi$ (right), the value of the action is $S=0.21472$, for the backward instanton, decreasing $\varphi$ by $2 \pi$ (left), it is $S=0.30969$. Hence, a noise induced average net rotation in the "forward direction" results in the weak noise limit, implying $\overline{\left\langle N_{z}\right\rangle}>0$ according to (24).

In fig. 22 the resulting instanton trajectories $\varphi^{*}(t)$ corresponding to backward and forward transitions are displayed. They show the qualitative behaviour discussed above. Note, however, that only the noise induced transition from the stable to the unstable orbit is shown. The complete transitions giving rise to $\varphi \mapsto \varphi \pm 2 \pi$ comprises also the subsequent relaxations from the unstable orbit to the next stable one. Consequently the stochastic transitions show substantially more structure than the rather poor caricatures used to represent them in Fig. [1.

The corresponding values of the action are $S=0.21472$ for the forward instanton increasing $\varphi$ by $2 \pi$ and $S=0.30969$ for the backward instanton decreasing $\varphi$ by $2 \pi$. Hence, at least in the weak noise limit, forward transitions are more likely (their action is smaller) than backward transitions and correspondingly for the parameter set specified in the caption of Fig. 2 we find a noise driven rotation in the direction of increasing $\varphi$. The mismatch between forward and backward transition clearly demonstrates the operation of the ratchet effect in our system. With the relevant transition probabilities being of order $\exp (-O(1) / D)$ the effect is however very weak and in particular rapidly disappears with $D \rightarrow 0$.

\section{B. Numerical solution of the FPE}

For the numerical solution of the FPE describing the complete two-dimensional dynamics for general values of the parameters it is convenient to expand the probability distribution $P(\theta, \varphi, t)$ in spherical harmonics $Y_{l}^{m}(\theta, \varphi)[51]$

$$
P(\theta, \varphi, t)=\sum_{l=0}^{\infty} \sum_{m=-l}^{l} A_{l, m}(t) Y_{l}^{m}(\theta, \varphi),
$$

with so far unknown time dependent complex expansion coefficients $A_{l, m}(t)$. Since $P(\theta, \varphi, t)$ is real we have

$$
A_{l,-m}(t)=(-1)^{m} A_{l, m}^{*}(t),
$$

with the star denoting complex conjugation. Moreover, from the normalization of $P(\theta, \varphi, t)$ we find

$$
A_{0,0}(t)=\frac{1}{\sqrt{4 \pi}} .
$$


Plugging the ansatz (54) into the FPE (47), exploiting (18), and using well-known properties of the spherical harmonics we find for $l \geq 1$ the following set of ordinary differential equations

$$
\begin{aligned}
\partial_{t} A_{l, m}(t)= & -\frac{H_{x}-i H_{y}(t)}{2}\left(h_{1}(l-1, m-1) A_{l-1, m-1}(t)+h_{2}(l+1, m+1) A_{l+1, m-1}(t)\right) \\
& +\frac{H_{x}+i H_{y}(t)}{2}\left(h_{1}(l-1,-m-1) A_{l-1, m+1}(t)+h_{2}(l+1,-m-1) A_{l+1, m+1}(t)\right) \\
& -D l(l+1) A_{l, m}(t)
\end{aligned}
$$

where we have introduced the auxiliary factors

$$
\begin{aligned}
& h_{1}(l, m)=(l+2) \sqrt{\frac{(l+m+1)(l+m+2)}{(2 l+1)(2 l+3)}} \\
& h_{2}(l, m)=(l-1) \sqrt{\frac{(l-m)(l-m-1)}{(2 l+1)(2 l-1)}} .
\end{aligned}
$$

Moreover, we find for the ensemble averages of the components of $\mathbf{e}(t)$ from (15)

$$
\begin{array}{r}
\left\langle e_{x}(t)\right\rangle=\langle\sin \theta(t) \cos \varphi(t)\rangle=-\sqrt{\frac{8 \pi}{3}} \Re A_{1,1}(t) \\
\left\langle e_{y}(t)\right\rangle=\langle\sin \theta(t) \sin \varphi(t)\rangle=\sqrt{\frac{8 \pi}{3}} \Im A_{1,1}(t) \\
\left\langle e_{z}(t)\right\rangle=\langle\cos \theta(t)\rangle=\sqrt{\frac{4 \pi}{3}} A_{1,0}(t)
\end{array}
$$

where $\Re z$ and $\Im z$ denote the real and imaginary part of the complex number $z$, respectively. For the $z$-component of the ensemble averaged but still time-dependent magnetic torque $\left\langle N_{z}\right\rangle$ this implies via (5)

$$
\left\langle N_{z}\right\rangle=-\sqrt{\frac{8 \pi}{3}}\left(H_{y}(t) \Re A_{1,1}(t)+H_{x} \Im A_{1,1}(t)\right) .
$$

We have used two somewhat different procedures to explicitly calculate this torque. The first is more general, the second is slightly more efficient for the special case of the time dependence (2).

In the first method we solve the system (57) of ordinary differential equations for the expansion coefficients $A_{l, m}(t)$ with $l \leq l_{\max }$ by a standard routine. We have used values of $l_{\max }$ up to 25 but found typically $5 \leq l_{\max } \leq 10$ to be sufficient. Starting with some initial condition, we integrate the equation until the periodic time dependence of the solution is reached, which was usually the case after at most two periods of the external field. We then integrate further for one period of the external driving, use the result for $A_{1,1}(t)$ in (63), and calculate the remaining time average numerically. Some care is needed here since this time-averaged torque $\overline{\left\langle N_{z}\right\rangle}$ is typically $2-3$ orders of magnitude smaller than the typical time-dependent values of $N(t)$. In the time average we are hence subtracting numbers of equal size from each other giving rise to well-known problems of numerical accuracy. The advantage of this method is that it works for any numerically sensible time dependence of the magnetic field $H_{y}(t)$ in (1).

The second method builds on the fact that the long-time solution of the FPE will be periodic in time with the period $2 \pi$ of the external driving. It is hence useful to expand the time-dependent expansion coefficients $A_{l, m}(t)$ in (54) into Fourier modes with respect to time

$$
A_{l, m}(t)=\sum_{s=-\infty}^{\infty} \tilde{A}_{l, m, s} e^{i s t}
$$

Instead of a system of ODE's for the coefficients $A_{l, m}(t)$ we find now from the FPE (47) a system of algebraic equations for the coefficients $\tilde{A}_{l, m, s}$. Moreover, the time averaged torque $\overline{\left\langle N_{z}\right\rangle}$ can be readily expressed as function of some of these coefficients by means of (63). The advantages of this method are that effectively we are directly dealing with the stationary solution of the FPE, i.e. no initial conditions are necessary and no equilibration process must be simulated, and that the time average of the torque needs not to be performed numerically. The disadvantage is that we have to specify $H_{y}(t)$ in order to find the algebraic system for the $\tilde{A}_{l, m, s}$ and that for general $H_{y}(t)$ this system will be rather dense with respect to the index $s$. 

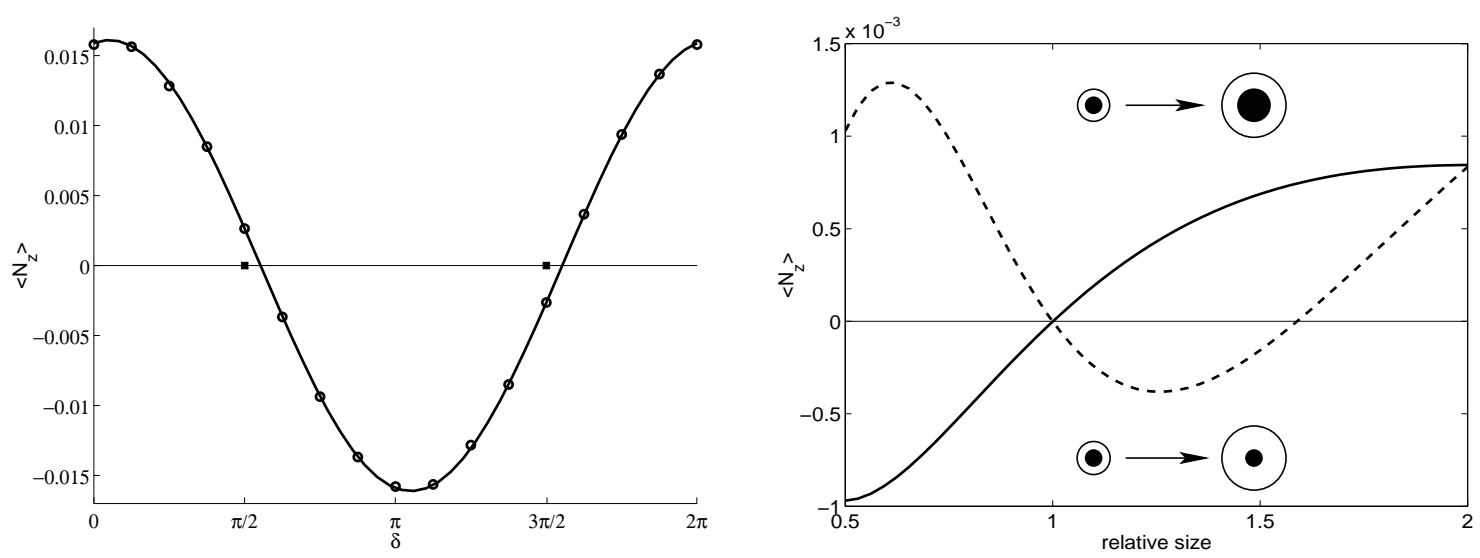

FIG. 3: Left: Averaged torque $\overline{\left\langle N_{z}\right\rangle}$ for the time modulation (2) with $H_{x}=0.3, H_{y}^{(1)}=H_{y}^{(2)}=1$ and $D=0.2$ as function of the phase angle $\delta$ as obtained from the numerical solution of the FPE (full line). Symbols are results from simulations of the Langevin equations (16) and (17) as reported in [30] with statistical errors being smaller than the symbol size. The squares on the horizontal axis are the points $(\pi / 2,0)$ and $(3 \pi / 2,0)$ at which the current reversal would occur in the reduced model (19). Right: Dependence of $\overline{\left\langle N_{z}\right\rangle}$ on the relative particle size for the same values of the parameters and $\delta$ fixed to $\delta_{0} \simeq 1.73$, the first point of current inversion in the left panel. The full line corresponds to a proportional change of the magnetic and hydrodynamic radii (top sketch), the dashed line is for a change of the hydrodynamic radius only (bottom sketch).

For the special time dependence (2) the situation is somewhat more gratifying since $H_{y}(t)$ involves only two Fourier components. Hence there are only couplings between coefficients $\tilde{A}_{l, m, s}$ differing in their $s$-value by at most 2 and the result for $\overline{\left\langle N_{z}\right\rangle}$ is a linear combination of just 5 different coefficients $\tilde{A}_{l, m, s}$. This makes the numerical analysis rather fast. Both methods must of course yield the same results when applied to the same $H_{y}(t)$. We have frequently used both to verify our numerical findings.

Using the numerical methods described above we have verified all the predictions derived in section ЏII on the basis of symmetry arguments. In particular we find $\overline{\left\langle N_{z}\right\rangle}=0$ if $H_{x}=0$ in accordance with (43) and $\overline{\left\langle N_{z}\right\rangle}=0$ for $H_{y}(t)=\cos (t)$, i.e. for (2) with $H_{y}^{(2)}=0$, or $H_{y}(t)=H_{y}^{(1)} \cos (t)+H_{y}^{(2)} \cos (3 t)$ in accordance with (44).

In Fig. [3 we give some examples for current inversions in our system. The left panel shows the averaged torque $\overline{\left\langle N_{z}\right\rangle}$ as function of the phase angle $\delta$ for a time dependent magnetic field $H_{y}(t)$ of the form (2). The results are consistent with (46). Moreover, the values of $\delta$ at which the torque changes sign are clearly different from $\pi / 2$ and $3 \pi / 2$ indicated by the squares on the horizontal axis. Hence the numerical solution verifies the statement of section that the current reversal in the full two dimensional model (16), (17) occurs at values of $\delta$ which are not fixed by any symmetry but rather depend in a complicated manner on all the other parameters of the problem.

This is further corroborated by the right panel of Fig. 3 depicting the torque $\overline{\left\langle N_{z}\right\rangle}$ as function of the relative particle size for $\delta=\delta_{0}$. There are two different ways to scale the particle size that make sense in a ferrofluid. In the first, both the ferromagnetic core of the particle as well as the polymer coating are scaled with the same factor. This gives rise to a proportional change of the magnetic moment $\mu$ and the particle volume $V$. In the dimensionless units adopted this implies unchanged fields $H_{x}$ and $H_{y}(t)$ and a noise intensity $D$ rescaled according to (14). In the other case, only the polymer coating is scaled and therefore only the hydrodynamic radius of the particle changes. Hence the magnetic moment remains the same whereas the volume $V$ changes. Correspondingly the dimensionless fields $H_{x}$ and $H_{y}(t)$ and the noise intensity $D$ change by the same factor. In view of (47) this can be absorbed in a rescaled time which in turn is equivalent to a changed frequency $\omega$ of the external field. As anticipated in section III we find current inversions in both cases.

\section{Effective field approximation}

It is very helpful to have some analytical expression for the average torque $\overline{\left\langle N_{z}\right\rangle}$ since the dependencies on the various parameters of the system can then be identified much more directly. In the present and subsequent subsections we discuss two approximate methods to calculate the time averaged torque in our system.

The first one employs the so-called effective field method to approximately solve the FPE. It has been a standard tool in the theory of ferrofluids for many years 52]. Two main ingredients are necessary. First, let us recall that the stationary (equilibrium) solution of the FPE (47) for a time independent homogeneous magnetic field $\mathbf{H}$ is of the form 


$$
P^{(0)}(\mathbf{e})=\frac{H}{4 \pi D \sinh (H / D)} \exp \left(\frac{\mathbf{e} \cdot \mathbf{H}}{D}\right),
$$

where $H$ denotes the modulus of the magnetic field, $H=|\mathbf{H}|$. Averages with this distribution are easily calculated, in particular we find the well-known result

$$
\langle\mathbf{e}\rangle^{(0)}=L(H / D) \mathbf{H} / H
$$

with the Langevin function

$$
L(x)=\operatorname{coth}(x)-1 / x .
$$

Second, for general, time-dependent fields $\mathbf{H}(t)$ the FPE (47) can be used to derive the exact equation

$$
\partial_{t}\langle\mathbf{e}\rangle+2 D\langle\mathbf{e}\rangle=-\langle\mathbf{e} \times(\mathbf{e} \times \mathbf{H})\rangle
$$

for the time evolution of the average $\langle\mathbf{e}\rangle$. Note that this equation for the first moment of $P(\mathbf{e}, t)$ has the usual flaw of being not closed but involving higher moments of the probability distribution.

The central idea of the effective field approximation is now to assume that the solution $P(\mathbf{e}, t ; \mathbf{H}(t))$ of the FPE for a general time dependent magnetic field $\mathbf{H}(t)$ can be written as equilibrium distribution for some a-priori unknown effective field $\mathbf{H}_{e}(t)$, i.e. $P(\mathbf{e}, t ; \mathbf{H}(t))=P^{(0)}\left(\mathbf{e} ; \mathbf{H}_{e}(t)\right)$. The average on the r.h.s. of (68) can then be performed and this equation gives rise to an evolution equation for the effective field $\mathbf{H}_{e}$ of the form [52]

$$
\partial_{t}\left(\frac{L\left(H_{e} / D\right)}{H_{e}} \mathbf{H}_{e}\right)=-2 D \frac{L\left(H_{e} / D\right)}{H_{e}}\left(\mathbf{H}_{e}-\mathbf{H}\right)-\frac{H_{e}-3 D L\left(H_{e} / D\right)}{H_{e}^{3}}\left(\mathbf{H}_{e} \times\left(\mathbf{H}_{e} \times \mathbf{H}\right)\right)
$$

Given $\mathbf{H}(t)$, this is a closed equation for the time evolution of $\mathbf{H}_{e}(t)$. Having obtained $\mathbf{H}_{e}(t)$ we get the desired approximate result for $\langle\mathbf{e}\rangle$ from (66)

$$
\langle\mathbf{e}\rangle_{e}=L\left(H_{e} / D\right) \mathbf{H}_{e} / H_{e}
$$

The solution of the partial differential equation (47) is hence replaced by the solution of the set of three coupled ordinary differential equations (69). There are other, equivalent forms of (69), see e.g. [53]. Exploiting (69), it is also possible to derive the following equivalent closed equation for $\langle\mathbf{e}\rangle[10]$

$$
\partial_{t}\langle\mathbf{e}\rangle_{e}=-\left(1-\frac{D\langle e\rangle_{e}}{H_{e}}\right)\left(\mathbf{H}_{e}-\mathbf{H}\right)+\left(1-3 D \frac{\langle e\rangle_{e}}{H_{e}}\right)\left(\frac{\langle\mathbf{e}\rangle_{e}}{\langle e\rangle_{e}} \cdot\left(\mathbf{H}_{e}-\mathbf{H}\right)\right) \frac{\langle\mathbf{e}\rangle_{e}}{\langle e\rangle_{e}},
$$

where $\langle e\rangle_{e}$ denotes the modulus of $\langle\mathbf{e}\rangle_{e}$ and the effective field $\mathbf{H}_{e}$ is to be expressed as function of $\langle\mathbf{e}\rangle_{e}$ by the inverse of (70). Note the difference between $\langle e\rangle_{e}=\left|\langle\mathbf{e}\rangle_{e}\right|$ and $\langle|\mathbf{e}|\rangle_{e} \equiv 1$.

In general, (69) can only be solved numerically. In Fig. 4 we give a comparison between results obtained in this way for the time dependence (2) and the corresponding outcome of the numerical solution of the FPE. It is clearly seen that the effective field approximation yields rather accurate results for the time dependent orientation of the particle and, equivalently, for the time dependent magnetization of the ferrofluid. On the other hand the results for the time averaged torque $\overline{\left\langle N_{z}\right\rangle}$ differ from the numerically exact values by a factor between 2 and 3 . The reason for this discrepancy lies in the fact that $\overline{\left\langle N_{z}\right\rangle}$ is typically 2-3 orders of magnitude smaller than typical values of the time dependent torque $\left\langle N_{z}\right\rangle$. In calculating the time average of the torque we hence subtract quantities of comparable order and so amplify the inaccuracies of the effective field approximation from a few percent to a few hundred percent.

In order to obtain an analytical expression for $\overline{\left\langle N_{z}\right\rangle}$ within the framework of the effective field approximation, it is useful to consider the case of small values of $H / D$ in which the Langevin function $L(x)$ or equivalently its inverse may be approximated by the first terms of the respective power series expansion. Using the lowest order approximation $L(x) \simeq x / 3$ we have $H_{e} \simeq 3 D\langle e\rangle_{e}$ and from (71) it then follows that

$$
\partial_{t}\langle\mathbf{e}\rangle_{e}+2 D\langle\mathbf{e}\rangle_{e}=2 \mathbf{H} / 3
$$

For large $t$ we therefore find

$$
\begin{aligned}
& \left\langle e_{x}\right\rangle_{e} \rightarrow \frac{2 H_{x}}{3 D} \\
& \left\langle e_{y}\right\rangle_{e} \rightarrow \frac{2 H_{y}^{(1)}}{3} \frac{2 D \cos t+\sin t}{4 D^{2}+1}+\frac{H_{y}^{(2)}}{3} \frac{D \sin (2 t+\delta)-\cos (2 t+\delta)}{D^{2}+1} .
\end{aligned}
$$



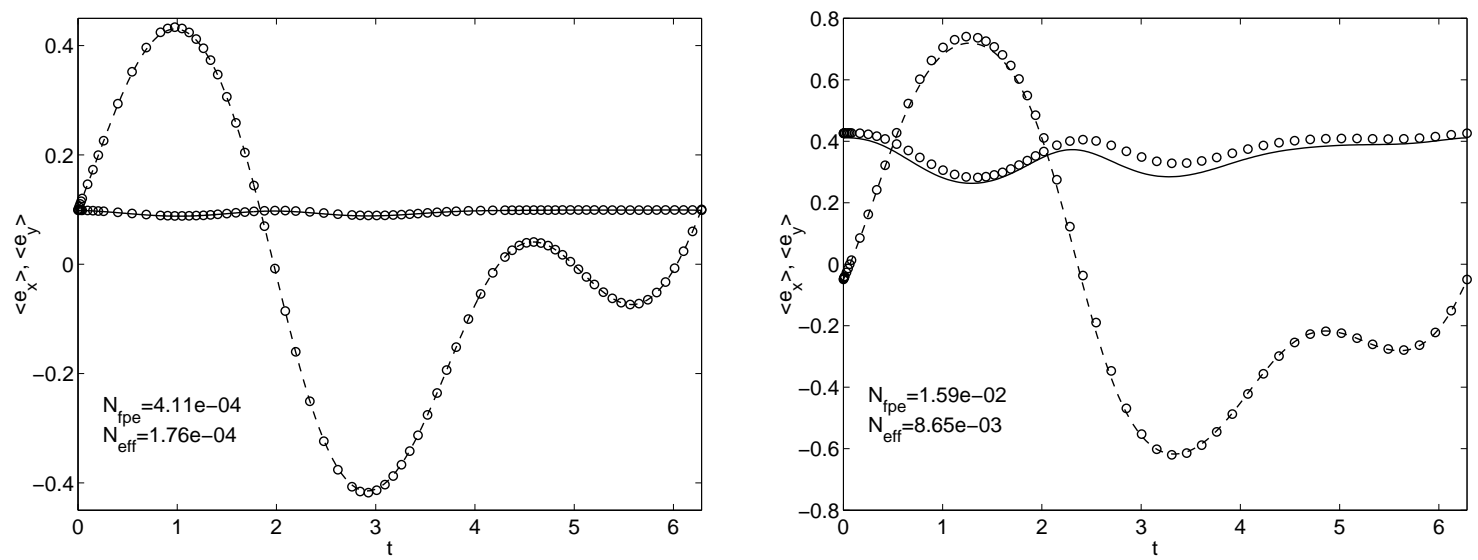

FIG. 4: Horizontal components of the average orientation $\langle\mathbf{e}\rangle$ of the particle as function of time for one period of the external magnetic field. The lines show the results for the $x$-and $y$-component of $\langle\mathbf{e}\rangle$ (full and dashed line, respectively) from the numerical solution of the Fokker-Planck equation (47) with a time dependence of the external field as given in (2). The symbols are the corresponding results from the effective field theory. The parameter values are $H_{x}=0.3, H_{y}^{(1)}=H_{y}^{(2)}=1, \delta=0$ as well as $D=1$ (left) and $D=0.2$ (right). The corresponding values for the time averaged torque $\overline{\left\langle N_{z}\right\rangle}$ are also displayed.

Hence $\left\langle e_{x}\right\rangle_{e}$ tends asymptotically to a constant whereas $\left\langle e_{y}\right\rangle_{e}$ contains only oscillating parts. From (5) we may thus infer that the torque $\left\langle N_{z}\right\rangle$ harmonically oscillates and therefore its time average vanishes, $\overline{\left\langle N_{z}\right\rangle}=0$.

To get a non-trivial result for $\overline{\left\langle N_{z}\right\rangle}$ we have to push the expansion of the Langevin function further. In other words, the non-linearity of the magnetization curve is essential to get a non-zero average torque. If the next term in the expansion is included, $L(x) \simeq x / 3-x^{3} / 45$, we already obtain an approximation for $L(x)$ that is accurate to within one per cent for the experimentally relevant values of $x$ which are generally less than 1 . Calculating the corrections to (73) and (74) induced by these higher order terms we finally find after some algebra the following result for the average torque

$$
{\overline{\left\langle N_{z}\right\rangle_{e}}}_{e}=\frac{H_{x}\left(H_{y}^{(1)}\right)^{2} H_{y}^{(2)}}{30} \frac{\cos \delta+2 D \sin \delta}{\left(1+D^{2}\right)\left(1+4 D^{2}\right)^{2}} .
$$

This result is in accordance with our symmetry considerations of section [II since it shows that both a non-zero field component in $x$-direction and an even higher harmonic in the time dependence of $H_{y}(t)$ are essential for a non-zero torque to occur, cf. (43), (444).

\section{Perturbative solution of the FPE}

In the last subsection we saw that the effective field approximation alone is not sufficient to get an explicit analytical expression for the time averaged torque $\overline{\left\langle N_{z}\right\rangle}$ in our system. Additionally we had to expand the Langevin function in its argument $H / D$ which amounts to a small field or equivalently large noise expansion. Moreover, the approximate result for the torque was typically at variance with the numerically exact result by a factor of about 2 .

It is therefore tempting to avoid the effective field approximation altogether and to use a perturbation expansion in the ratio of magnetic field strength and noise intensity right in the Fokker-Planck equation (47). This is conveniently accomplished by making the formal substitution $\mathbf{H} \mapsto \epsilon \mathbf{H}$ in (47) and by expanding the solution of the FPE in a power series in $\epsilon$

$$
P(\theta, \varphi, t)=P^{(0)}(\theta, \varphi, t)+\epsilon P^{(1)}(\theta, \varphi, t)+\epsilon^{2} P^{(2)}(\theta, \varphi, t)+\epsilon^{3} P^{(3)}(\theta, \varphi, t)+\ldots .
$$

At the end of the calculation $\epsilon$ is put equal to one. It will turn out that we have to use the expansion up to third order in $\epsilon$ to get a non-zero result for the average torque $\overline{\left\langle N_{z}\right\rangle}$. Since the potential $U(\theta, \varphi, t)$ is of order $\epsilon$ we find in zeroth order

$$
\lim _{t \rightarrow \infty} P^{(0)}(\theta, \varphi, t)=\frac{1}{4 \pi}
$$


describing the stationary distribution of pure rotational diffusion. Using the expansion (76) in (47) we get a system of linear inhomogeneous partial differential equations for the various $P^{(n)}(\theta, \varphi, t)$ that can be solved in principle one after the other.

This procedure is, however, rather cumbersome. It can be somewhat simplified by using the exact equation (68), the r.h.s of which is of order $\epsilon$. Hence to find $\langle\mathbf{e}\rangle$ from this equation to order $\epsilon^{n}$ it is sufficient to calculate the average on the r.h.s. with an expression for $P(\theta, \varphi, t)$ accurate to order $\epsilon^{(n-1)}$. In our case the lowest order non-zero result for $\overline{\left\langle N_{z}\right\rangle}$ is $\mathcal{O}\left(\epsilon^{4}\right)$, hence we need $\langle\mathbf{e}\rangle$ to order $\epsilon^{3}$ (cf. (5), (15)) and therefore the second order result for $P(\theta, \varphi, t)$ would be sufficient.

It is, however, more convenient to use again the expansion (154) of $P(\theta, \varphi, t)$ in terms of spherical harmonics. The perturbation ansatz (76) then translates into an expansion of the coefficients $A_{l, m}(t)$ in powers of $\epsilon$

$$
A_{l, m}(t)=A_{l, m}^{(0)}(t)+\epsilon A_{l, m}^{(1)}(t)+\epsilon^{2} A_{l, m}^{(2)}(t)+\epsilon^{3} A_{l, m}^{(3)}(t)+\ldots
$$

The ODE's (57) couple only coefficients $A_{l, m}(t)$ which differ in both $l$ and $m$ by 1 . Moreover, we know from (56) $A_{0,0}(t)=1 / \sqrt{4 \pi}=\mathcal{O}(1)$ and hence $A_{l, m}(t)=\mathcal{O}\left(\epsilon^{l}\right)$. As shown by (63) the torque is determined by $A_{1,1}(t)$ alone. We hence need an expression for $A_{1,1}(t)$ correct up to order $\epsilon^{3}$.

To this end we determine from (57) and (56) first the $\mathcal{O}(\epsilon)$ terms of $A_{1, m}(t)$. With these we calculate the necessary $A_{2, m}(t)$ to order $\epsilon^{2}$ which in turn are used to determine the $\mathcal{O}\left(\epsilon^{3}\right)$ terms in $A_{1,1}(t)$. The procedure is sketched in fig. 5 . Note that it is sufficient to calculate coefficients $A_{l, m}(t)$ with $m \geq 0$ because of the symmetry property (55).

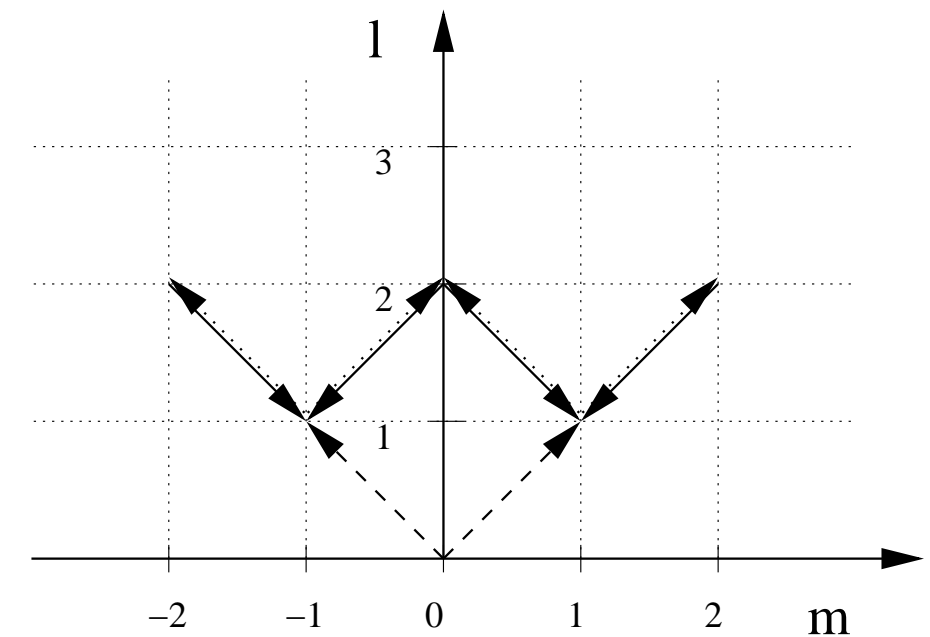

FIG. 5: Diagrammatic sketch of the perturbative determination of the expansion coefficients $A_{l, m}$ in (54). As shown by the arrows only $A_{l, m}$ differing in both $l$ and $m$ by 1 are coupled by equations (57). In a first step we start from $A_{0,0}$ as given in (56) and obtain the $\mathcal{O}(\epsilon)$ terms of $A_{1, \pm 1}$ (dashed arrows). Next $A_{2,0}$ and $A_{2, \pm 2}$ are determined to order $\epsilon^{2}$ (dotted arrows). Finally these are used to get the $\mathcal{O}\left(\epsilon^{3}\right)$ contributions to $A_{1,1}$ (full arrows) from which the torque can be extracted to order $\epsilon^{4}$ using (63). All other $A_{l, m}$ are irrelevant for $\overline{\left\langle N_{z}\right\rangle}$ at this order in $\epsilon$. Note also that the left half of the diagram is redundant due to the symmetry property (55).

In this way we first get from the $\mathcal{O}(\epsilon)$ equations

$$
\begin{aligned}
& \left(\partial_{t}+2 D\right) A_{1,1}^{(1)}(t)=-\frac{1}{\sqrt{6 \pi}}\left(H_{x}-i H_{y}(t)\right) \\
& \left(\partial_{t}+2 D\right) A_{1,0}^{(1)}(t)=0
\end{aligned}
$$

Since we are interested in the asymptotic solution valid for large $t$ we can put $A_{1,0}^{(1)}(t) \equiv 0$. Next we use the $\mathcal{O}\left(\epsilon^{2}\right)$ equations

$$
\begin{aligned}
\left(\partial_{t}+6 D\right) A_{2,2}^{(2)}(t) & =-\frac{3}{\sqrt{5}}\left(H_{x}-i H_{y}(t)\right) A_{1,1}^{(1)}(t) \\
\left(\partial_{t}+6 D\right) A_{2,0}^{(2)}(t) & =\sqrt{\frac{6}{5}}\left(H_{x} \Re A_{1,1}^{(1)}(t)-H_{y}(t) \Im A_{1,1}^{(1)}(t)\right)
\end{aligned}
$$



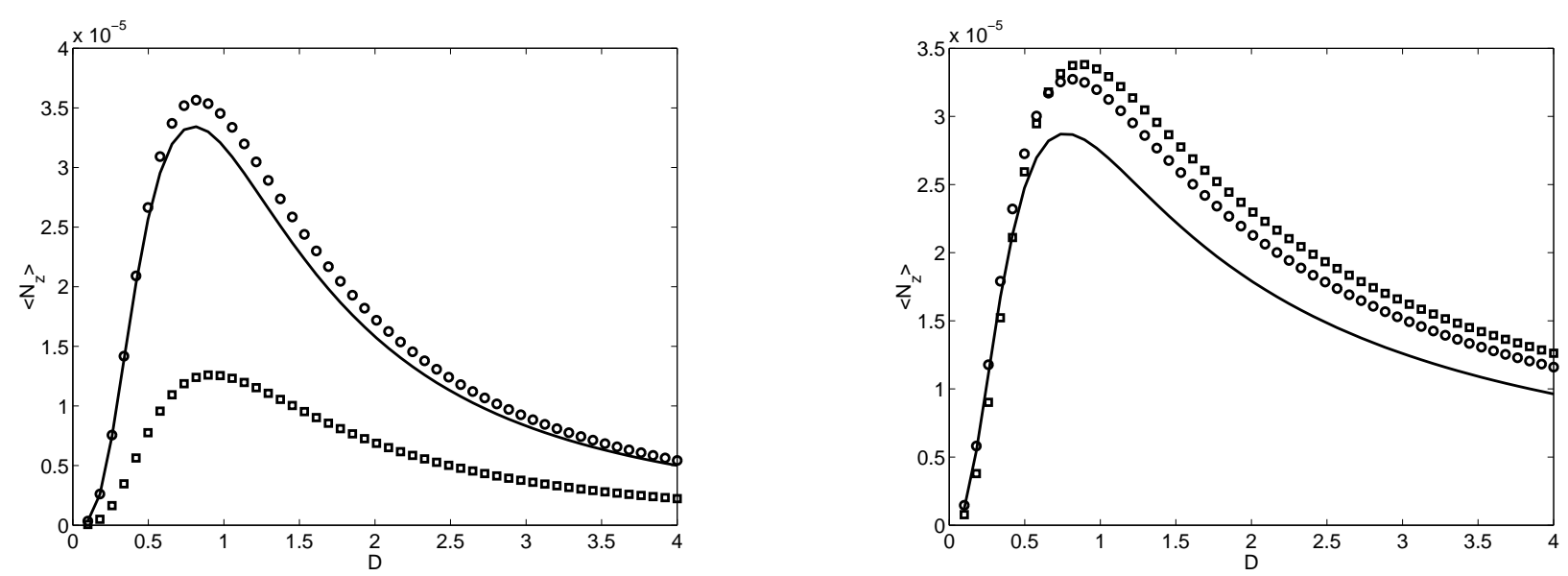

FIG. 6: Left: Time averaged torque $\overline{\left\langle N_{z}\right\rangle}$ as a function of the noise intensity $D$ for the time dependence (2) of the magnetic field. The parameter values are $H_{x}=0.5 D, H_{y}^{(1)}=0.5 D, H_{y}^{(2)}=0.15 D$, and $\delta=0$. The scaling of the magnetic field strength with $D$ ensures that the perturbation parameter $\epsilon$ stays constant. Shown are the perturbative results (80) (circles) and (75) (squares) together with the numerically exact result (full line). Right: Same for the time dependence (3) of the oscillating field with $H_{y}^{(0)}=H_{y}^{(1)}=0.5 D$. The approximate results are given by (82) and (81), respectively.

to determine the relevant $A_{2, m}(t)$ to the desired order. Finally we obtain $A_{1,1}^{(3)}(t)$ from

$$
\left(\partial_{t}+2 D\right) A_{1,1}^{(3)}(t)=-\frac{1}{\sqrt{30}}\left(H_{x}-i H_{y}(t)\right) A_{2,0}^{(2)}(t)+\frac{1}{\sqrt{5}}\left(H_{x}+i H_{y}(t)\right) A_{2,2}^{(2)}(t) .
$$

The explicit expressions for the various coefficients $A_{l, m}^{(n)}(t)$ are rather long, the above system of equations is therefore conveniently solved with the help of a computer algebra. Focusing on the specific time dependent field $H_{y}(t)$ from (2), exploiting (63) and performing the time average over one period of the external driving we finally get the following lowest order perturbative result for the average torque

$$
{\overline{\left\langle N_{z}\right\rangle_{p}}}_{p}=\frac{H_{x}\left(H_{y}^{(1)}\right)^{2} H_{y}^{(2)}}{40} \frac{9\left(1+29 D^{2}\right) \cos \delta+2 D\left(1+99 D^{2}\right) \sin \delta}{\left(1+D^{2}\right)\left(1+4 D^{2}\right)\left(1+9 D^{2}\right)\left(1+36 D^{2}\right)} .
$$

The dependencies on the parameters $H_{x}, H_{y}^{(1)}$, and $H_{y}^{(2)}$ are identical to those of the effective field result (75), whereas the dependence on the noise strength $D$ is more complicated. One readily verifies that the symmetry properties (35)(38) are satisfied by (80). In particular, these symmetries imply that the torque $\overline{\left\langle N_{z}\right\rangle}$ must to be an odd function of both the static field and the oscillating field. Moreover, symmetry reasons also imply [15] that in linear order of the oscillating field one cannot expect a finite $\overline{\left\langle N_{z}\right\rangle}$. Rather, a coupling of several modes of the oscillating field (harmonic mixing) is required, see also [37, 44, 46]. All together, these arguments explain that the first nontrivial contribution must be (at least) of fourth order in the magnetic field strength and that within a linear response theory one will always find $\overline{\left\langle N_{z}\right\rangle}=0$ 54. On the other hand this does of course not imply that the non-linear magnetization curve alone would "explain" the effect. To understand the microscopic origin of the angular momentum transfer from the external field to the particle one really has to go to the description in terms of the FPE as discussed above, see also [55, [56].

In the left panel of Fig. 6 we compare the two approximate expressions (75) and (80) for the averaged torque with the results obtained from the numerical solution of the FPE. Displayed is the dependence of the torque on the noise intensity $D$ which is different in the two approximations. In order to keep the expansion parameter $\epsilon \sim H / D$ roughly constant when changing $D$ we have scaled the magnetic fields strengths with $D$. As can be seen, up to values of $\epsilon \simeq 0.5$ the accuracy of (80) is rather good whereas (75) describes the torque only qualitatively.

Proportional change of magnetic field strength and noise intensity is equivalent to a change of the frequency $\omega$ of the external field in (11). Fig. [6 hence also demonstrates the resonance-like character of the investigated ratchet effect. $\overline{\left\langle N_{z}\right\rangle}$ is largest when the deterministic time scale of the external driving matches an intrinsic stochastic time scale of the system related to the Brownian relaxation time $\tau_{B}$ defined in (13).

The two approximate expressions for the torque (75) and (80) differ in their dependence on the phase angle $\delta$. When using (80) to fit the experimental results reported in [10] we find for the Brownian relaxation time the value 
$\tau_{B} \simeq 6.410^{-4} \mathrm{~s}$ instead of $\tau_{B} \simeq 1.810^{-3} \mathrm{~s}$ as obtained on the basis of (75) in [10]. The experiments were done with a ferrofluid with $\eta \simeq 0.1$ Pas. Using (13) the two results for $\tau_{B}$ hence translate in fits for the particle diameter of $d \simeq 26 \mathrm{~nm}$ and $d \simeq 36 \mathrm{~nm}$, respectively. These values exceed the typical diameter of roughly $10 \mathrm{~nm}$ by factors between 2 and 3, respectively. The main reason for this discrepancy is probably the polydispersedness of real ferrofluids, having a particle size distribution with a long tail (see e.g. [3], ch.2). Hence, a whole spectrum of relaxation times is necessary to accurately describe the dynamics of the magnetization. However, this issue is not at the focus of the present investigation. We also note that the transferred torque typically increases with the particle size and hence the described ratchet effect is likely to be dominated by the larger grains in the ferrofluid.

A similar approximate calculation of the average torque is also possible for the time dependence (3). The result of the effective field approximation is

$$
{\overline{\left\langle N_{z}\right\rangle_{e}}}_{e}=\frac{2}{45} \frac{H_{x} H_{y}^{(0)}\left(H_{y}^{(1)}\right)^{2}}{D\left(4 D^{2}+1\right)^{2}}
$$

whereas we find from the perturbative solution of the FPE

$$
{\overline{\left\langle N_{z}\right\rangle_{p}}}_{p}=\frac{H_{x} H_{y}^{(0)}\left(H_{y}^{(1)}\right)^{2}}{30} \frac{44 D^{2}+3}{D\left(4 D^{2}+1\right)^{2}\left(36 D^{2}+1\right)} .
$$

The two expression only differ significantly from each other if the noise intensity $D$ is very small. In the right panel of Fig. 6 they are compared with the result from the numerical solution of the FPE (47). For $\epsilon \simeq 0.5$ the accuracy is again seen to be rather satisfactory

\section{CONCLUSIONS}

In the present paper we have theoretically investigated the rotational Brownian motion of colloidal ferromagnetic particles in an oscillating magnetic field. The central tool was the Fokker-Planck equation for the probability density $P(\mathbf{e}, t)$ of the particle orientation e at time $t$. Solving this equation either numerically or approximately by using the effective field approximation as well as a perturbative expansion we have determined the time averaged torque $\overline{\left\langle N_{z}\right\rangle}$ exerted by the magnetic field on the particles. The main and a priori quite unexpected qualitative finding is the fact that a purely oscillating magnetic field without net rotating component can transfer angular momentum to a ferromagnetic grain. As basic mechanism behind this transfer, a ratchet effect was identified by which the magnetic field rectifies the thermal fluctuations of the particle orientation that arise due to random collisions with the molecules of the carrier liquid. The detailed operation of this ratchet effect in the present system was discussed on the basis of a weak-noise analysis of the Fokker-Planck equation for a closely related one-dimensional system in section IV A

Via the viscous coupling to the carrier liquid the torque on the particle is transmitted to the liquid. The combined microscopic torques of the huge number of individual nanoparticles then yields a macroscopic rotation of the ferrofluid as a whole, as observed experimentally in [10]. In the absence of thermal noise, no average torque can arise, i.e. thermal fluctuations are an indispensable requirement for the rotation of the individual grains and hence of the ferrofluid as a whole to occur.

The results from the Fokker-Planck equation are completely consistent with rather general symmetry considerations detailed in section III Moreover, they agree very well with simulations of the corresponding Langevin equations as given in [30] and quantitatively describe the experimental findings reported in [10].

We remark that our present system puts forward a new type of thermal ratchet device which does not fit into any previously known specific class of ratchet systems. Adopting the classification scheme from [15], our present system has some similarity with so-called asymmetrically tilting ratchets as well as with a so-called traveling potential ratchets, however in the generalized sense involving two counterpropagating traveling potentials. Yet there remain significant differences with both these classes. In particular, we note that we are not dealing here with a periodic, asymmetric so-called "ratchet"-potential. Rather, at any fixed instance of time the relevant potential is perfectly symmetric about the instantaneous direction of the magnetic field and hence no preferential direction of rotation seems to exists. It is only via the time evolution that a symmetry breaking arise, which is sometimes called a dynamical symmetry breaking.

In summary we hope that the present investigation has demonstrated that ferrofluids are very suitable systems to study various aspects of thermal ratchet behaviour, and that it may stimulate further theoretical, numerical and experimental work in this direction. 


\section{Acknowledgments}

Special thanks is due to Ubbo Felderhoff for several interesting suggestions and to Martin Raible for providing the numerical simulation results in Fig. 3. Discussions with Hanns Walter Müller, Konstantin Morozov, and Mark Shliomis are gratefully acknowledged. Part of this work was done during a sabbatical stay of A.E. at the Laboratoire de Physique Théorique at the Université Louis Pasteur in Strasbourg. He acknowledges the kind hospitality as well as financial support from the Volkswagenstiftung. This work was further supported by Deutsche Forschungsgemeinschaft under SFB 613 and RE 1344/3-1 and by the ESF-program STOCHDYN.

[1] A. Einstein, Ann. d. Physik 19, 371 (1906)

[2] M. I. Shliomis, Sov. Phys. Usp. 17, 153 (1974)

[3] R. E. Rosensweig, Ferrohydrodynamics, (Cambridge University Press, Cambridge, 1985)

[4] J.-C. Bacri, A. Cebers, and R. Perzynski, Phys. Rev. Lett. 72, 2705 (1994)

[5] A. V. Lebedev, A. Engel, and K. I. Morozov, New. J. Phys. 5, 57 (2003)

[6] J. P. McTague, J. Chem. Phys. 51, 133 (1969)

[7] M. I. Shliomis and K. I. Morozov, Phys. Fluids 6, 2855 (1994)

[8] F. Gazeau et al., Europhys. Lett. 35, 609 (1996)

[9] H. W. Müller, Phys. Rev. Lett. 82, 3907 (1999)

[10] A. Engel, H. W. Müller, P. Reimann, and A. Jung, Phys. Rev. Lett. 91, 060602 (2003)

[11] J. C. Maxwell, Theory of Heat (Longmans, Green, and Co., London, 1871)

[12] M. v. Smoluchowski, Z. Physik 13, 1069 (1912)

[13] R. P. Feynman, R. B. Leighton, and M. Sands, The Feynman Lectures on Physics-Vol.1, ch. 46 (Addison-Wesley, Reading, 1963)

[14] S. L. Harvey and A. F. Rex (eds.), Maxwell's Demon: Entropy, Information, Computing (Adam Hilger, Bristol, 1990)

[15] P. Reimann, Phys. Rep. 361, 57 (2002)

[16] R. D. Astumian and P. Hänggi, Physics Today 55, 33 (2002)

[17] M. O. Magnasco, Phys. Rev. Lett. 71, 1477 (1993)

[18] F. Jülicher, A. Ajdari, and J. Prost, Rev. Mod. Phys. 69, 1269 (1997)

[19] J. Rousselet, L. Salome, A. Ajdari, and J. Prost, Nature 370, 446 (1994)

[20] H. Linke (ed.) Special issue on ratchets and Brownian motors: Basic experiments and applications, Appl. Phys. A 75, 167-352 (2002)

[21] H. Linke et al., Science 286, 2314 (1999)

[22] I. Turcu and C. M. Lucaciu, J. Phys. A: Math. Gen. 22, 995 (1989)

[23] P. Palffy-Muhoray and E. Weinan, Mol. Cryst. Liq. Cryst. 320, 193 (1998)

[24] S. Flach, A. E. Miroshnichenko, and A. A. Ovchinikov, Phys. Rev. E65, 104438 (2002)

[25] W. T. Coffey, Y. P. Kalmykov, and J. T. Waldron, The Langevin equation with applications in physics, chemistry and electrical engineering, (World Scientific, Singapore, 1996)

[26] F. Ritort, Phys. Rev. Lett. 80, 6 (1998)

[27] Z. Wang, C. Holm, and H. W. Müller, Phys. Rev. E66, 021405 (2002)

[28] L. D. Landau and E. M. Lifshitz, Electrodynamics of Continuous Media, 2nd ed., § 34 (Pergamon, New York, 1984).

[29] L. D. Landau and E. M. Lifshitz, Fluid Mechanics, 2nd ed., § 20, (Pergamon, New York, 1987).

[30] M. Raible and A. Engel, Langevin Equation for the Rotation of a Magnetic Particle, to appear in Applied Organometallic Chemistry

[31] H. Risken, The Fokker-Planck Equation, Springer, Berlin 1984.

[32] V. Becker and A. Engel, unpublished

[33] P. Curie, J. Phys. (Paris) 3. Série (théorique et appliqué) t. III, 393 (1894).

[34] A. Ajdari, D. Mukamel, L. Peliti, and J. Prost, J. Phys. I France 4, 1551 (1994)

[35] R. Kanada and K. Sasaki, J. Phys. Soc. Jpn. 68, 3759 (1999)

[36] S. Weiss, D. Koelle, J. Müller, K. Barthel, and R. Gross, Europhys. Lett. 51, 499 (2000).

[37] S. Flach, O. Yevtushenko, and Y. Zolotaryuk, Phys. Rev. Lett. 84, 2358 (2000)

[38] S. Cilla and L. M. Floria, Phys. Rev. E 63, 031110 (2001)

[39] E. Neumann and A. Pikovsky, Eur. Phys. J. B 26, 219 (2002)

[40] O. Yevtushenko, S. Flach, Y. Zolotaryuk, and A. A. Ovchinikov, Europhys. Lett. 54, 141 (2001)

[41] B. Yan, R. M. Miura, and Y.-D. Chen, J. Theor. Biol. 210, 141 (2001)

[42] P. Reimann, Phys. Rev. Lett. 86, 4992 (2001)

[43] S. Denisov, S. Flach, A. A. Ovchinikov, O. Yevtushenko, and Y. Zolotaryuk, Phys. Rev. E 66, 041104 (2002)

[44] K. Seeger and W. Maurer, Solid State Commun. 27, 603 (1978)

[45] D. R. Chialvo and M. M. Millonas, Phys. Lett. A209, 26 (1995)

[46] I. Goychuk and P. Hänggi, Europhys. Lett. 43, 503 (1998) 
[47] R. P. Feynman, A. R. Hibbs, Quantum Mechanics and Path Integrals (McGraw-Hill, New York, 1965)

[48] L. S. Schulman, Techniques and Applications of Path Integration (Wiley, New York, 1981)

[49] M. Chaichian and A. Demichev, Path Integrals in Physics, Vol.1 (IOP Publishing, Bristol, 2001)

[50] J. Lehmann, P. Reimann, and P. Hänggi, Phys. Rev. Lett. 84, 1639 (2000); Phys. Rev. E62, 6282 (2000); phys. stat. sol. (b) 237, 53 (2003) and further references therein.

[51] M. Abramowitz and I. Stegun (eds.), Handbook of Mathematical Functions (National Bureau of Standards, Washington D.C., 1964)

[52] M. A. Martsenyuk, Yu. L. Raikher, and M. I. Shliomis, Sov. Phys. JETP 38, 412 (1974)

[53] H. W. Müller and M. Liu, Phys. Rev. E64, 061405 (2001)

[54] T. Alarcon, A. Perez-Madrid, J. M. Rubi, Phys. Rev. Lett. 85, 3995 (2000)

[55] M. Shliomis, Phys. Rev. Lett. 92, 188901

[56] A. Engel and P. Reimann, Phys. Rev. Lett. 92, 188902 